\title{
12
}

\section{Fuzzy Logic for controlling call admission in ATM networks}

\author{
M. Fontaine and D.G. Smith
}

University of Strathclyde

Department of Electronic \& Electrical Engineering

204 George Street

Glasgow G1 1XW

United Kingdom

Tel: +44 1415482090

Fax: +441415524968

E-mail:m.fontaine@eee.strath.ac.uk

\begin{abstract}
An essential function of ATM is Connection admission control (CAC). To achieve higher network utilisation, bursty calls are statistically multiplexed on a common link. We present a new CAC scheme based on fuzzy logic (FL) and artificial neural networks (ANNs) to exploit this key feature of ATM networks. Defining a hybrid intelligent system enables us to take advantage of both the learning capabilities of ANNs and the interpretability properties of FL. The ANN is used in the learning phase to automatically tune the fuzzy system (i.e. to define the fuzzy rules and the membership functions) whilst during the control phase, the fuzzy system forecasts the QoS values. The CAC procedure is able to determine on line the cell loss probability (CLP) that a connection will exhibit when accepted into the ATM network. Because of its possible hardware implementation as well as its adaptive and learning capabilities, this scheme constitutes a good candidate for a robust real-time CAC.
\end{abstract}

\section{Keywords}

ATM networks, CAC, Fuzzy Logic, Artificial Neural Networks. 


\section{INTRODUCTION}

Telecommunications networks are growing rapidly and becoming more and more complex. In (Reeve, 1996), Reeve makes the point: "the problem is how to effectively manage and control such networks since users expect an ever increasing quality of service, and these services are becoming critical for the successful day to day operation of businesses".

To cope with this growing complexity and to prevent an overwhelming cost associated with it, telecommunications network management (TNM) systems have to undergo some radical changes. First, a high level of automation is required to process the huge amount of data available to the TNM system. Since a part of these data is often incomplete, uncertain and sometimes conflicting, human interaction remains essential when classical techniques are used (Muller, 1993). To prevent the network from being increasingly dependent on highly qualified network operators (Reeve, 1996), artificial intelligence (AI) based techniques may be considered. These techniques present $a$ priori two advantages: they are able to deal with incomplete and even incoherent data, and they limit the need of human interaction.

As an example, the next section will concentrate on one particular telecommunications application and the possible use of AI techniques.

\section{CAC IN ATM NETWORKS}

ATM is the transmission technology chosen for the future broadband integrated services digital network (B-ISDN). ATM-based networks are capable of supporting a wide range of telecommunications services with stringent quality of service (QoS) specifications. These benefits, however, come at a price; contrary to a common opinion, ATM is a complex technology whose management constitutes a set of difficult problems. This subject encompasses both traffic control and congestion control, which could be basically described as preventive and reactive control. We are interested in traffic control, and more especially in the call admission control (CAC) process. This function is vital in an ATM network since it decides whether or not a new connection should be accepted into the network. A connection is accepted when sufficient resources are available to carry the connection at the quality of service (QoS) required by the user through the whole network, without altering the QoS of existing connections.

One key feature of ATM is the statistical multiplexing of bursty connections which allows the network to achieve higher utilization (Roberts, 1991). Although many CAC schemes have been proposed in the literature (Fontaine, 1996b), none 
was deemed accurate and fast enough to be an adequate candidate to deal with variable bit rate (VBR) traffic. Most of the time, they provide solutions based on assumptions. These assumptions rely either on the assumed traffic model (which is supposed to be Gaussian, Interrupted Poisson Process, etc.) or on the approximation of an exact mathematical formula. Obviously, each assumption imposes restrictions on the scheme. These techniques are not flexible enough to support ATM traffic. They are designed for a known and determined situation, but they are not really suitable for an ATM network which is supposed to carry both current and unknown future services. It seems extremely complex to devise a controller using the classical methods, to solve this multi-objective optimization problem. To cope with this difficulty, a new kind of approach based on "intelligent" techniques could be considered. In this paper, we claim that the use of AI based techniques provides efficient, effective, flexible and robust control. Since it is difficult for a network to acquire complete statistics of the input traffic, it has to make a decision based on incomplete information. Hence, the decision process is full of uncertainty (Cheng, 1996). Because fuzzy logic has been applied to time-varying and ill-defined problems, we decided to consider this technique.

\section{FUZZY LOGIC AND CAC}

Fuzzy logic takes decisions based upon a set of rules written in an English-like language (Zadeh, 1973). Its main feature is the ability to model a complex system without requiring a functional input-output description. Using fuzzy logic allows us to overcome the mathematical complexity of the problem. As a matter of fact, it encodes the expert knowledge about ATM CAC without requiring a model for the actual CAC mechanism. Moreover, an important benefit is that fuzzy logic deals with situations in which no rule is defined (unlike expert systems). The essential steps in the design of a fuzzy system are to define the fuzzy variables (including the choice of the variables and of their membership functions), the fuzzy rules and the defuzzification method (see Figure 1).

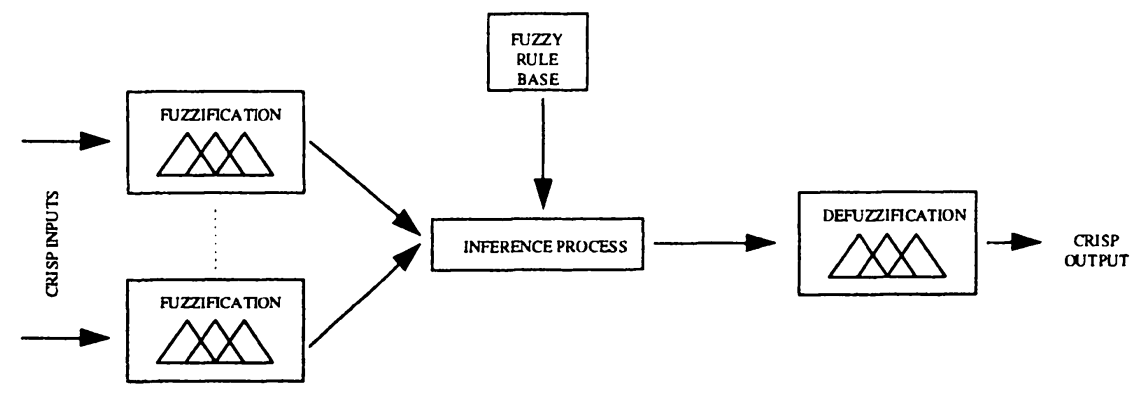

Figure $1 \mathrm{~A}$ fuzzy inference system. 
The fuzzification process requires membership functions to translate crisp inputs into degrees of membership. In most cases, the membership function has either a triangular or trapezoidal shape, but in order to get a continuous and differentiable function, we consider a bell-shaped function in the form of equation (1).

$$
f(x)=\frac{1}{1+\left(\frac{x-c}{a}\right)^{2 b}}
$$

$a$ controls the width of the curve, $b$ determines how flat the curve is at its top, and $c$ represents the location of the centre.

Previous studies (Elwalid, 1995) and (Mitrou, 1994) have shown that three parameters are important where statistical multiplexing is concerned. These parameters are the peak bit rate to link capacity ratio, the activity factor (meanto-peak ratio) and the mean load (sum of the mean bit rates of accepted connections divided by the link capacity). These three quantities constitute the inputs. There is no specific rule to determine the number of membership functions per input. In this paper, like in (Fontaine, 1996a), the trial and error method led us to choose three membership functions per input.

The fuzzy rule base contains fuzzy "if-then" rules. Different forms of fuzzy ifthen rules have been presented in the literature so far (Mendel, 1995) and (Jang, 1992). In this study, we use the form proposed by Takagi and Sugeno (Takagi, 1983), which has fuzzy sets involved only in the premise part. The conclusion part of each rule is a linear combination of the input variables plus a constant term. For a two-input fuzzy system, a rule looks like: "If $i p 1$ is Fuzzy-set1 and $i p 2$ is Fuzzy-set2 then output $=p * i p 1+q * i p 2+r$ ".

The defuzzification performs the reverse operation from the fuzzification and it delivers a crisp output. To follow the Takagi and Sugeno model, the output is taken to be the weighted average of each rule output.

To optimize the fuzzy controller and to prevent the designer from tuning its system with the unsatisfactory trial and error method (Kosko, 1992), a modified algorithm coming from neural network tools, the error back propagation algorithm (Nauck, 1993); is used to define the fuzzy rules and to automatically generate the membership functions (i.e. to set the parameters $(a, b, c)$ ). Based on this model, we designed a "neuro-fuzzy" system able to determine a priori and on line the cell loss probability (CLP) a connection will exhibit when accepted into the ATM network. The fuzzy system is actually embedded in an artificial neural

\footnotetext{
* These features will be required later on to apply the back-propagation learning algorithm.
} 
network framework which provides adaptive and learning capabilities. The next section shows some results of the experiments performed.

\section{SIMULATION}

A fuzzy system has been designed to forecast the cell loss probability experienced by VBR sources multiplexed in a 48 cell buffer switch with a 155.52 $\mathrm{Mb} / \mathrm{s}$ output link capacity. The system has been trained with the cell loss results obtained by the RACE (Research on Advanced Communications in Europe) consortium R2061 (Exploit) (Witters, 1994). Six different traffic sources (modelled as on-off sources) were considered (cf. Table 1).

Table 1 Traffic types

Traffic Peak bit rate (Mb/s) Mean bit rate (Mb/s) Mean burst size (cells)

$\begin{array}{llll}\text { A.3.1 } & 31.1 & 6.22 & 1467\end{array}$

$\begin{array}{llll}\text { A.3.2 } & 31.1 & 1.56 & 734\end{array}$

$\begin{array}{llll}\text { B.3.1 } & 7.78 & 3.89 & 917\end{array}$

$\begin{array}{llll}\text { B.3.2 } & 7.78 & 0.39 & 183\end{array}$

$\begin{array}{llll}\text { C.3.1 } & 1.94 & 0.97 & 229\end{array}$

$\begin{array}{llll}\text { C.3.2 } & 1.94 & 0.39 & 92\end{array}$

A total of 14 traffic scenarios have been studied: 6 homogeneous traffic scenarios (cf. Table 1) and 8 heterogeneous traffic mixes. In the heterogeneous case, two traffic types are multiplexed together on the same link. Note that the measurements correspond to the overall cell loss ratio and not the cell loss ratio individual sources or traffic types experience.

If only the three inputs proposed in the previous section are considered then the buffer in the switch is not taken into account. Depending on the ratio between the buffer size and the mean burst size, the buffer may absorb whole bursts thereby preventing the cell loss probability from being degraded. The three-input-model was satisfactory when the system was trained only with the bufferless convolution 
algorithm (Fontaine, 1996a) but, it becomes obsolete when real traffic is used for training. A fourth input corresponding to the ratio between the buffer size and the mean burst size is considered to take into account the buffer's influence (Roberts, 1991) and (Boyer, 1995). Both approaches (3- and 4-input system) have been developed and trained with the same set of items coming from measurements. Each system has been trained with the backpropagation algorithm for 100 epochs (i.e. the training set has been used 100 times).

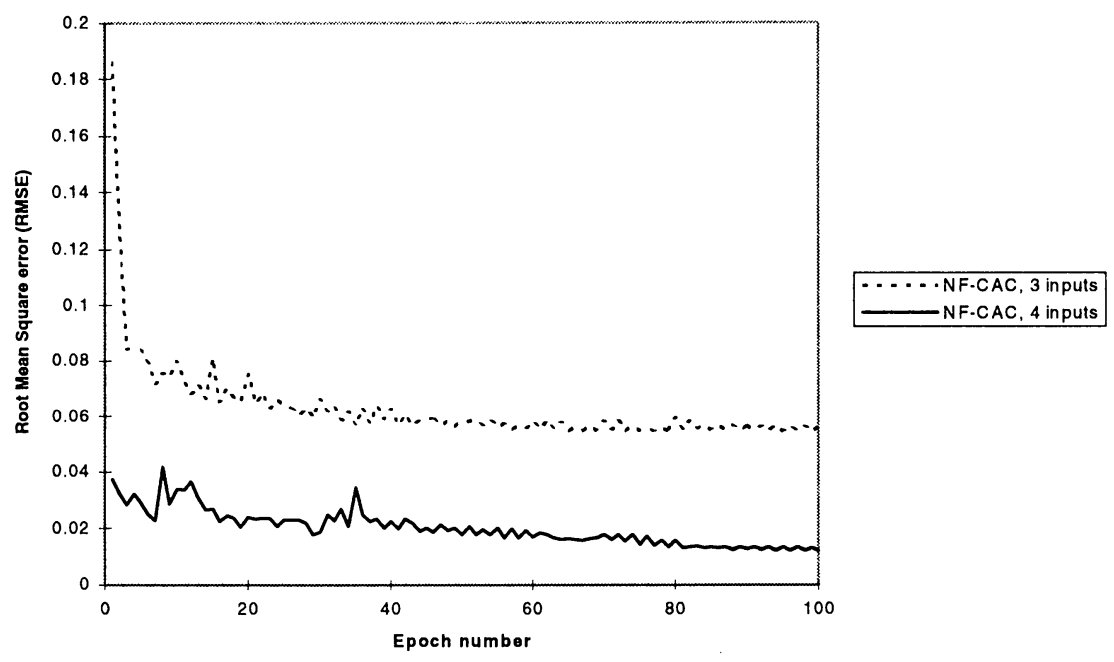

Figure 2 Error during the training epochs.

At the end of the training phase, the parameter set leading to the minimal root mean square error (RMSE) is kept and the system is ready to operate in running phase. Figure 2 displays the RMSE for both systems versus the epoch number. It is clear that the 4-input-system has a more adequate set of inputs since the RMSE is much lower. Actually, all the figures in Table 2 are lower in the case of a 4input-system. The a priori knowledge given to the second system through the 4 inputs is more suitable for the fuzzy CAC approach (Fontaine, 1997). 
Table 2 Training errors

\begin{tabular}{lllll}
\hline Nb of inputs & RMSE min & RMSE max & RMSE mean & RMSE std dev. \\
\hline 3 & 0.054331 & 0.18643 & 0.062811 & 0.016038 \\
4 & 0.012168 & 0.041867 & 0.019996 & 0.006384 \\
\hline
\end{tabular}

From now onwards, the 4-input-system is considered. The parameters (i.e. membership function parameters $(a, b, c)$ and fuzzy rule parameters) have been tuned during the training phase. The set of parameters chosen is the one leading to the minimal RMSE which has been reached at the 100th epoch. Some typical results of the running phase are displayed in Figures 3 (homogeneous case) and 4 (heterogeneous case). In Figure 4, the number of A.3.1 sources is constant and equal to 2, whilst the number of C.3.1 sources varies. For comparison, the results obtained by analytical approach (convolution algorithm) are displayed on the same figures. The results obtained from the neuro-fuzzy system are close to the measurements obtained from the test-bed.

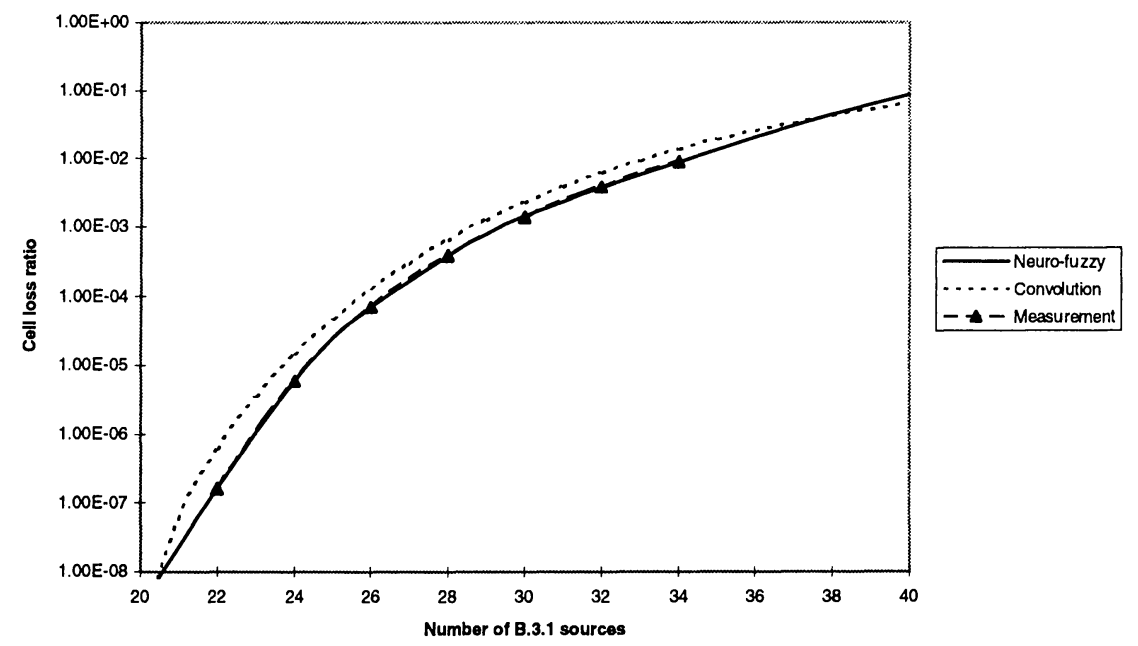

Figure 3 Homogeneous case. 


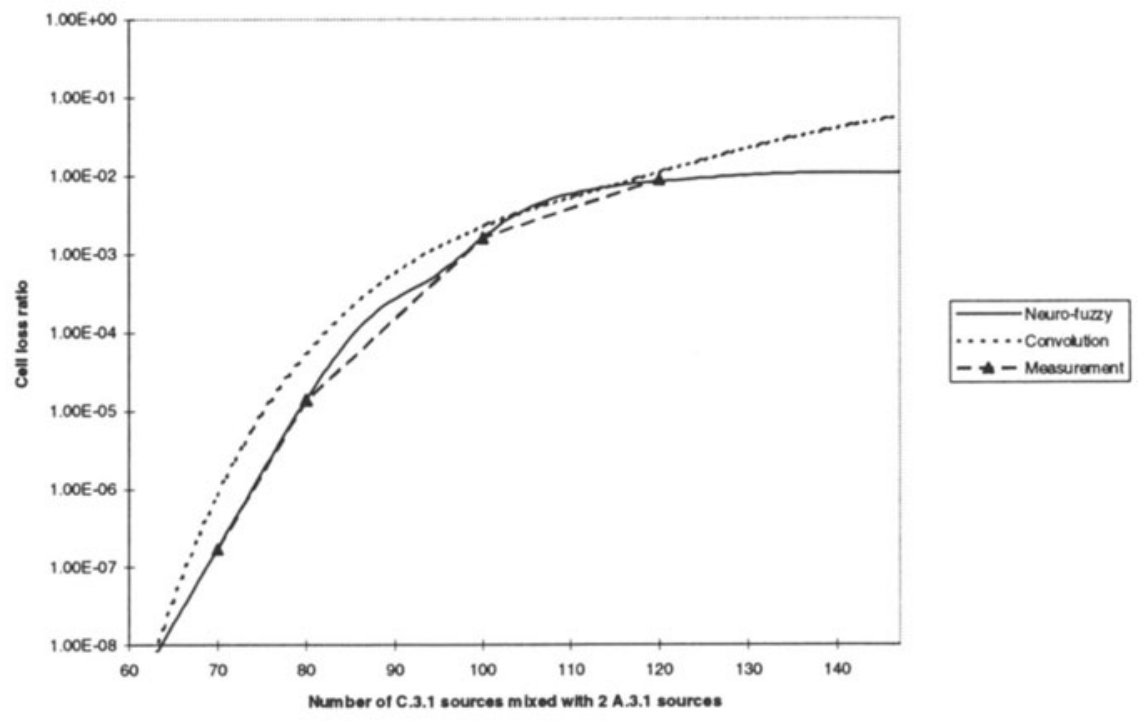

Figure 4 Heterogeneous case.

The neuro-fuzzy system is very sharp for the situations for which it has been trained. Hence, the difference between the predictions of the fuzzy system and the actual measurements from the test-bed is almost null. On average, over the whole training set, the error between prediction and measurement (which is actually the minimal training error) is just over $1 \%$. Moreover, it should be noticed that the system is able to interpolate and its behaviour between two distinct measurements (training points) is satisfactory. In the homogeneous case, the neuro-fuzzy system gives a very good response. Its predictions are better than the ones obtained by the convolution algorithm which is based on a bufferless model. Note that for the traffic mix displayed (cf. Figure 4), there are only 4 training points and the system is still able to give a good answer over a large area. Some oscillations may be noticed in this latter case, meaning that the training set was too small . Even though, the results obtained demonstrate that fuzzy logic and neural networks constitute an interesting and suitable alternative to analytical approaches for CAC. The next stage is to increase the number of training items by using an on-line training. Using both batch and on-line learning prevents from having long batch learning periods and enables the system to acquire new and updated information about the actual traffic. An on-line learning algorithm is now under study. 


\section{CONCLUSION}

In this paper, we have argued that $\mathrm{AI}$ is going to be used in the coming years to manage and control telecommunications networks. These techniques are actually needed to cope with the growing complexity of communications networks. Of course, any AI technique is not suitable for every management task. Depending on the requirements, one specific technique or a sub-set of AI techniques should be considered. To highlight this point, we have presented a case study: a fuzzy logic based algorithm for connection admission control in ATM networks. In order to automate the design process of the fuzzy controller, the self-learning capabilities of neural networks has been considered. The results show that the combination of these two techniques are promising. The on-line learning capability is another appealing feature which will be considered for further study.

\section{REFERENCES}

Boyer, J. Gravey, A. and Sevilla, K. (1995) Resource allocation for worst case traffic in ATM networks. In First Workshop on ATM Traffic Management WATM'95, Paris, pages 3-19.

Cheng, R.-G. (1996) Design of a fuzzy traffic controller for ATM networks. IEEE/ACM Transactions on Networking, 4(3):460-469.

Elwalid, A. Mitra, D. and Wentworth, R.H. (1995) A new approach for allocating buffers and bandwidth to heterogeneous, regulated traffic in an ATM node. IEEE JSAC, 13(6):1115-1127.

Fontaine, M and Smith, D.G. (1996a) A neuro-fuzzy approach to connection admission control in ATM networks. In IEE Thirteenth UK teletraffic symposium, Glasgow.

Fontaine, M and Smith, D.G. (1996b) Bandwidth allocation and connection admission control in ATM networks. Electronics and Communication Engineering Journal, 8(4):156-164.

Fontaine, $M$ and Smith, D.G. (1997) Intelligent techniques and telecommunications networks. In European Symposium on Intelligent Techniques, Bari, Italy.

Jang, J.-S.R. (1992) Self-learning fuzzy controllers based on temporal back propagation. IEEE Transactions on Neural Networks, 3:714-723. 
Kosko, B. (1992) Neural Networks and Fuzzy Systems: a dynamical systems approach to machine intelligence. Prentice-Hall International Editions.

Mendel, J.M. (1995) Fuzzy logic systems for engineering: a tutorial. Proceedings of the IEEE, 83(3):345-377.

Mitrou, N.M Kontovasilis, K.P. Kroner, H. and Iversen, V.B. (1994) Statistical multiplexing, bandwidth allocation strategies and connection admission control in ATM networks. European Transactions on Telecommunications, 5(2):161-175.

Muller, C. Magill, E.H. Prosser, P. and Smith, D.G. (1993) Artificial intelligence in Telecommunications. In IEEE Globecom'93, Houston.

Nauck, D. and Kruse, R. (1993) A fuzzy neural network learning fuzzy control rules and membership functions by fuzzy error backpropagation. In IEEE ICNN'93, San Francisco.

Reeve, J.M. and Mars, P. (1996) A review of non-symbolic artificial intelligence techniques for network management and control. In IEE Thirteenth UK teletraffic symposium, Glasgow.

Roberts, J.W. (1991) Variable-Bit-Rate traffic control in B-ISDN. IEEE Communications Magazine, pages 50-56.

Takagi, T and Sugeno, M. (1983) Derivation of fuzzy control rules from human operator's control actions. In Proc. of the IFAC Symp. on Fuzzy Information, Knowledge Representation and Decision Analysis, pages 55-60.

Witters, J. Nielsen, A.B. Elvang, R. Kroeze, J. Petterson, H. Aarstad, E. and Renger, T. (1994) Results of experiments on traffic control using real applications. Technical report, Race project R 2061

Zadeh, L.A. (1973) Outline of a new approach to the analysis of complex systems and decision processes. IEEE transactions on Systems, Man, and Cybernetics, 3(1):28-44. 